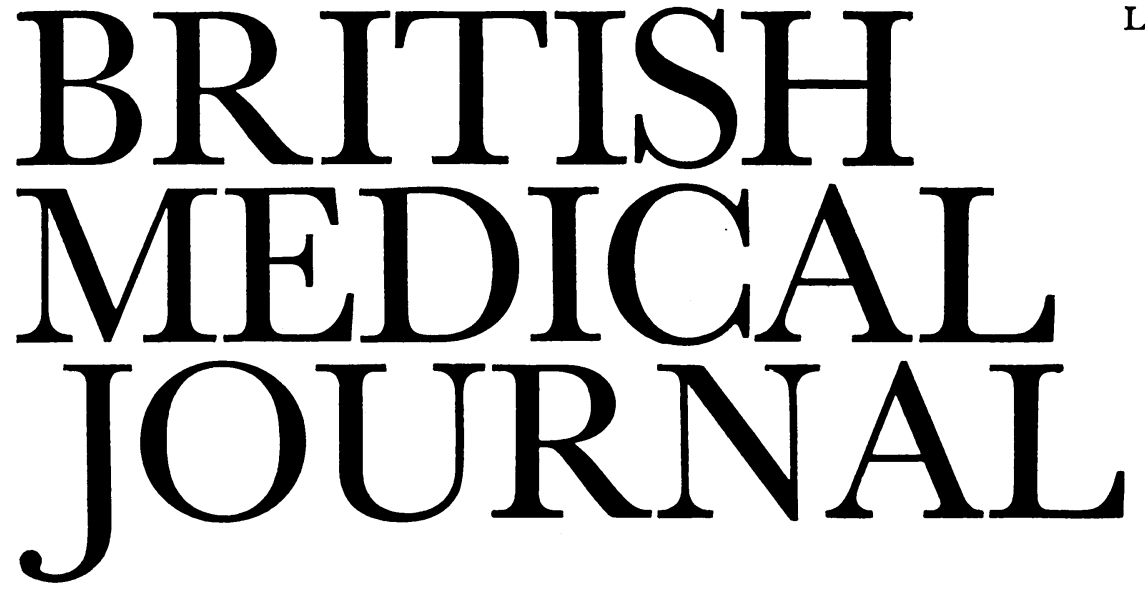

\title{
Congenital cataract: a cause of preventable blindness in children
}

A child with congenital cataracts needs surgical treatment by the age of 8 weeks if the eyes are to function even reasonably well. The management is difficult-for the doctor, the parents, and the child-but the enhanced visual result in most cases justifies the difficulties and the risks. All those concerned in the medical care of young children need, therefore, to be aware of the features of congenital cataracts and the importance of prompt referral to the ophthalmologist.

Early treatment has become mandatory for congenital cataract as the result of several developments in the last 15 years. Firstly, ophthalmologists have become aware of the fundamental importance of amblyopia in the management of visual disorders in childhood. ${ }^{1}$ In amblyopia central vision is lost because of some defect in the eye during a sensitive period of development of the visual system. The results of studies in animals strongly suggest that in human infancy there may be a period of grace of up to 2 months of age when the effects of visual deprivation are of less consequence because they are less profound and reversible. ${ }^{2} 3$ After this come 6 to 18 months of increased sensitivity-the human equivalent of the laboratory animal's critical period-and then a gradually declining sensitivity for several years, so that by the age of 9 or 10 a child's visual system is no longer affected by degraded visual input.

Amblyopia may be caused not only by opacity in the optical media of the eye-such as cataract-but also by a blurred retinal image such as occurs in uncorrected aphakia (the absence of the lens from its normal site with the loss of its refractive role). Our greater understanding of the mechanisms of amblyopia has had two effects on the management of cataract: most children with congenital cataract who require surgery in infancy should have that surgery completed by 2 months of age; and early accurate correction of aphakia is essential if a sharp retinal image is to be produced during the sensitive period.

Important changes have also occurred in the surgical treatment of congenital cataracts, ${ }^{4}$ substantially improving the short-term and long-term prognosis. Extended-wear contact lenses are now available for correcting aphakia in infants. These lenses are readily fitted without anaesthesia, and infants tolerate them well despite the high refractive error. Aphakia cannot be corrected satisfactorily by spectacles because these require very high power lenses and so are impracticably heavy and optically unsatisfactory. Intraocular lens implants are not recommended for infants at present.

How, then, should congenital cataract be recognised? An infant with a unilateral cataract will have a white pupil and a small eye, or he may have a unilateral squint noticed by parents or relatives. With bilateral congenital cataracts parents may notice early on that their baby does not seem to look at them, and they may also notice white pupils. By the age of 3 months a baby with severe bilateral cataracts will start to develop nystagmoid movements on attempted fixation, and this, or an associated squint, is generally the mode of presentation in what are now regarded as late cases.

Clinical diagnosis is generally made initially by the paediatrician or general practitioner who listens to the mother's worries about the baby's vision, the appearance of the pupils, or the presence of nystagmus or squint. Careful inspection of the eyes may show the small eye or the white pupil, but the absence of a white pupil does not exclude a cataract since the opacity may be situated posteriorly in the lens. One useful technique is to examine the eye with an ophthalmoscope as though to look at the fundus but keeping about $30 \mathrm{~cm}$ away; the absence of a red reflex indicates an opacity in the media of the eye. Closer inspection with the ophthalmoscope is possible if the lens system of the ophthalmoscope is set at +20 dioptres. Such a quick and simple examination should form part of the routine screening of infants. If a cataract is suspected, referral to an ophthalmologist should be arranged urgently, especially if the baby is less than 1 year old.

A search for the cause of the cataract is important but that should not delay the management of the cataract itself. Investigations should be carried out after consultation between ophthalmologist and paediatrician, each of whom will be able to exclude certain causes on clinical grounds, thus saving time and money on unnecessary tests.

Sometimes the decision whether or not a cataract requires treatment may be difficult. The optical disadvantages of aphakia, combined with the risks and possible failure of treatment, make it inadvisable to operate and start optical treatment unless it is certain that the child will be visually handicapped unless he is treated. Most cases fall clearly into the "obviously blind" or "obviously sighted" categories, but 
the management of borderline cases may be determined by a variety of techniques for testing vision. Most doctors managing such children, however, rely on behavioural observations in borderline cases. If the infant fixes objects steadily without nystagmus or squinting and responds to subtle clues-such as a silent smile or movements of small objects-the vision is unlikely to be sufficiently poor to warrant early treatment. When there is any doubt little is lost by reassessing the child after an interval of one or two weeks. In borderline cases repeated examinations may be needed to make this critical decision.

Bilateral congenital cataract is the most common cause of potentially treatable blindness in infancy. Almost certainly early surgery leads to better visual results-provided that optical correction is started promptly. ${ }^{6}$ Unfortunately, delays in diagnosis and referral result in most babies with bilateral severe congenital cataracts being seen by the ophthalmologist for the first time between 2 and 4 months of age. Effects of visual deprivation must be expected by the age of 4 months, and ideally all patients should be referred early enough for surgery and optical correction to be completed, if necessary, by the age of 2 months.

After surgery the baby is fitted with high gas-transfer contact lenses designed for continuous wear. ${ }^{7}$ The risk of corneal damage from these is substantial, and frequent follow-up visits are necessary. The contact lenses are not strictly necessary once the child reaches 3 years or so, when he can wear spectacles.

The cost of contact lens treatment (to both the parents and the NHS) is admittedly high-perhaps between $£ 200$ and $£ 1200$ a year, depending on the numbers of lenses used and the distance from the contact lens department. Nevertheless, if the improvement in the child's visual performance is sufficient to shift him from one educational category to another such treatment is clearly cost effective.

Until recently the visual prognosis for eyes with unilateral cataract was universally agreed to be so poor that surgery was indicated only for cosmetic reasons or to prevent glaucoma resulting from a "hypermature" lens. Recently Beller et al have described a series of children who were operated on and optically corrected within days of birth, followed by vigorous occlusion of the normal eye. Careful monitoring of the vision of both eyes by psychophysical or electrophysical means showed that nine patients who had received ideal treatment had Snellen acuities of about 6/9. The social relevance of extended, and possibly risky, treatment for a non-blinding condition is, however, a matter for debate.

The notion that in infants with congenital rubella surgery for cataract was best delayed for some months or even years because of the risk of endophthalmitis has now been outdated by improved surgical technique, and bilateral cataracts due to rubella require early surgery like any others. The indications for surgery are probably even stronger in unilateral rubella cataracts because of the substantial risk of late visual deterioration due to macular degeneration. ${ }^{9}$

N S C RICE

Consultant Ophthalmic Surgeon,

Moorfields Eye Hospital,

London EC1V 2PD

D TAYLOR

Consultant Ophthalmic Surgeon,

Hospital for Sick Children,

London WCIN 1EH

1 Wiesel TN, Hubel DH. Effects of visual deprivation on morphology and physiology of cells in the cats lateral geniculate body. $f$ Neurophysiol 1963;26:978-93.
2 Vaegan, Taylor D. Critical period for deprivation amblyopia in children. Trans Ophthalmol Soc UK 1979;99:432-9.

${ }^{3}$ Awaya S. Stimulus vision deprivation amblyopia in humans. In: Reineceke $\mathrm{RD}$, ed. Strabismus. Proceedings of the third meeting of the International Strabismological Association. New York: Grune and Stratton, 1978:31.

4 Rice NSC. Lens aspiration-a decade in retrospect. Trans Ophthalmol Soc UK 1977;97:48-51.

5 Taylor D. Choice of technique in surgery for congenital cataract. Tran Ophthalmol Soc UK 1981;101:114-7.

6 Taylor D, Vaegan, Morris JA, Rogers JE, Warland J. Amblyopia in bilateral infantile and juvenile cataract. Relationship to timing of treatment. Trans Ophthalmol Soc UK 1979;99:170-5.

${ }^{7}$ Morris J, Taylor D, Rogers JE, Vaegan, Warland J. Contact lens treatment of aphakic infants and children. Fournal of the British Contact Lens Association $1979 ; 2: 22-30$.

${ }^{8}$ Beller R, Hoyt CS, Marg E, Odom JV. Good visual function after neonatal surgery for congenital monocular cataracts. Am $\mathcal{f}$ Ophthalmo $1981 ; 91: 559-65$.

${ }^{9}$ Frank KE, Punell EW. Subretinal neovascularization following rubella retinopathy. Am $\mathcal{F}$ Ophthalmol $1978 ; 86: 462-6$.

\section{Emporiatrics-travellers' health}

Good health is everyone's major source of wealth; yet most people, paradoxically, do nothing about their health until they lose it. This is especially true of people who live and travel abroad-yet they suffer many more health problems than comparable populations who stay at home. One survey of a large group of American travellers found that a quarter had had one or more symptoms of illness while travelling ${ }^{1}$; a comparable survey of Swiss travellers found that threequarters had had symptoms. ${ }^{2}$ The Conference Board, an American non-profit business research institute, reviewed the experiences of 30 major corporations and compared the quality of medical care received by corporate employees going abroad (a privileged group of travellers) to a game of Russian roulette.

Emporiatrics (Greek emporos one who goes on shipboard as a passenger +iatrike medicine) is the term coined to describe the science of the health of travellers. ${ }^{3}$ Travellers face special health risks. Firstly, they are subject to disorders induced by rapid changes of environment such as upsets in the circadian rhythms, motion sickness, and diarrhoea; secondly, in developing countries they are exposed to infectious diseases that do not exist in their home countries such as malaria, giardiasis, and dengue; and, thirdly, they are separated from familiar and accessible sources of medical care. Nevertheless, the lure of travel seems undiminished. Indeed, Mackay recently pointed out that never before in history have so many people travelled and have people travelled so far or so fast. The latest available statistics of the International Civil Aviation Organisation shows that there were nearly 900 million domestic and international passengers on scheduled and unscheduled airline services in $1980 .^{5}$ To this group of business men and tourists must be added the hundreds of thousands of immigrants, refugees, and migrant labourers who frequently travel by other means. Meeting the health needs of these travellers who are moving rapidly between countries and continents is a responsibility shared by the medical profession, by the travellers themselves, by travel organisations, by airline and shipping companies, and by host governments. ${ }^{6}$ The new science of emporiatrics can point the way towards the development of policies and 